\title{
Interference Mitigation and Mobility Management for D2D Communication in LTE-A Networks
}

\author{
Ravindra S ${ }^{a}$, Dr. Siddesh G K ${ }^{\mathrm{b}}$ \\ ${ }^{a}$ Research Scholar, Dept. of ECE, DSCE, K.S.Layout, Bengaluru, India \\ ${ }^{b}$ Professor, Dept. of ECE, JSSATE, Uttarahalli-Kengeri Rd, Bengaluru, India
}

Received: 11 October 2018; Accepted: 16 February 2019; Published: 08 March 2019

\begin{abstract}
Interference and Mobility management are the main parameters which affect the signal capacity and reduce the transmission efficiency for D2D communication in LTE-A networks. When the users are in the same spectrum, due to the coexistence of D2D pairs and cellular users interference management becomes a critical issue. In this paper, we introduce an interference management algorithm that maximizes the performance of D2D communication in LTE-A networks for both uplink and downlink transmissions. Optimal routing selection techniques reduce the total path distance which helps to minimize the interference and improve the overall network capacity. Mobility management is also a challenging issue in IP-mobile networks. When two DUEs change their locations from one BS to the other, handover delay calculation becomes very important to maintain the communication without interruption. In this paper we show power control techniques which helps to reduce the effect of interference and also we addressed a method for the lower delay under complex mobility issues with uninterrupted D2D communication.
\end{abstract}

Index Terms: Device to Device Communication, Interference management, SINR, Mobility management, Handover delay.

(C) 2019 Published by MECS Publisher. Selection and/or peer review under responsibility of the Research Association of Modern Education and Computer Science

\section{Introduction}

Device-to-device (D2D) communications allow user equipments to communicate directly with each other. Interference mitigation is a major issue for efficient D2D communication. Due to the coexistence of device to device pairs and cellular users, the D2D pairs generate effective interference towards base stations and cellular

* Corresponding author. Tel.: +91 8553634644

E-mail address: ravindra-ece@dayanandasagar.edu 
users. The interference to the D2D pairs significantly reduces the efficiency of the D2D communication. Channel assignment and power control algorithm techniques helps to mitigate the interference in D2D communication. High throughput can be obtained by properly adjusting the transmission power of all the nodes.

Resource allocation schemes to decrease the complexity of the network and to find interference limited areas are proposed in [2]. In these methods the cell is divided into two parts, the inner region which is near to eNB and the outer region which is away from eNB. However, there are no power control techniques to increase the performance of devices in the outer region. In these techniques since the cellular users do not share the same resources with device to device pairs, these schemes cannot be applied to the heavily deployed device to device pairs [15]. Fractional frequency reuse (FFR) is a scheme that reuses the same frequency in cellular networks to improve network capacity and spectral efficiency [1]. Interference alignment (IA) scheme used to reduce the interference effect [4]. But it requires global channel state information (CSI) to mitigate interference in the entire system. User selection methods are introduced to improve the sum rate [11]. However, it is constrained to the users with a single antenna. Hence the most effective technique to address interference mitigation is power control method [3]. In this method, a predefined maximum power level is calculated to control the interference and to guarantee the QoS requirement for D2D communication. Power reduction in turn maximizes the capacity of network. Two power control techniques are proposed in this paper. Femto cell and macro cell cellular networks are considered for the interference management in the first method. In the second method power requirements are calculated based on the distance between D2D users and CUEs.

When two mobile nodes are in communication they may change their locations and hence mobility management becomes a major issue. In the cellular mode, it is not a big task to maintain the services uninterrupted $[6,10]$. But in D2D mode it becomes a great challenge for mobility management since a proper algorithm is yet to be developed to maintain the communication without interruption. Handover delay (HD) for reliable communication between two user equipments while moving is a serious issue and a solution is shown in the proposed technique.

Following the introduction, this paper is organized as follows. In Section 2 the system model is described. Section 3 describes interference and mobility management. Section 4 presents simulation parameters and results. Section 5 concludes the paper.

\section{Related Work}

Interference reduces the capacity and transmission efficiency in D2D communication. Some of the interference types which impact on D2D communication are: Inter-symbol interference (ISI), Co-channel interference (CCI), Adjacent channel interference (ACI) and Cross-tier interference [12].

The disturbance of a signal with the other signals during communication is called Inter symbol interference. Co-channel interference appears between the two access points which use same frequency. Adjacent-channel interference is due to the extra power of the signal from the next channel. Cross-tier interference is the interference from one tier to another.

The interference appears in D2D communication is broadly divided into: Interference from D2D communication to cellular communication and Interference between D2D pairs.

Interference greatly reduces the efficiency, throughput and also other factors related to the D2D communication. The eNBs which shares channels in both uplink (UL) and downlink (DL) phases are contrived by the interference. Hence channel assignment and power control algorithms place an important role to mitigate interference problem in D2D communication [13]. The throughput and channel capacity can be increased by controlling the transmission power of all eNBs and CUs in LTE-A networks [15]. The D2D pair that reuses the channel is placed far away from the cellular user to avoid co-channel interference. Fig. 1 shows the interference problems between D2D pairs and CUs, where many DUEs coincide with a CUE in single cell. The channel state information can be obtained for all UEs when DUEs uses uplink channel resources at the eNBs. Because of multiple DUEs, cross-tier interference can be seen at the eNB. Hence it is to be greatly avoided to minimize 
the interference effect on the CUE. The proposed power control algorithm measures and reduces the power levels of DUE, CUE and eNBs based on distance. Decision of eNB usage is considered based on the distance between UEs.

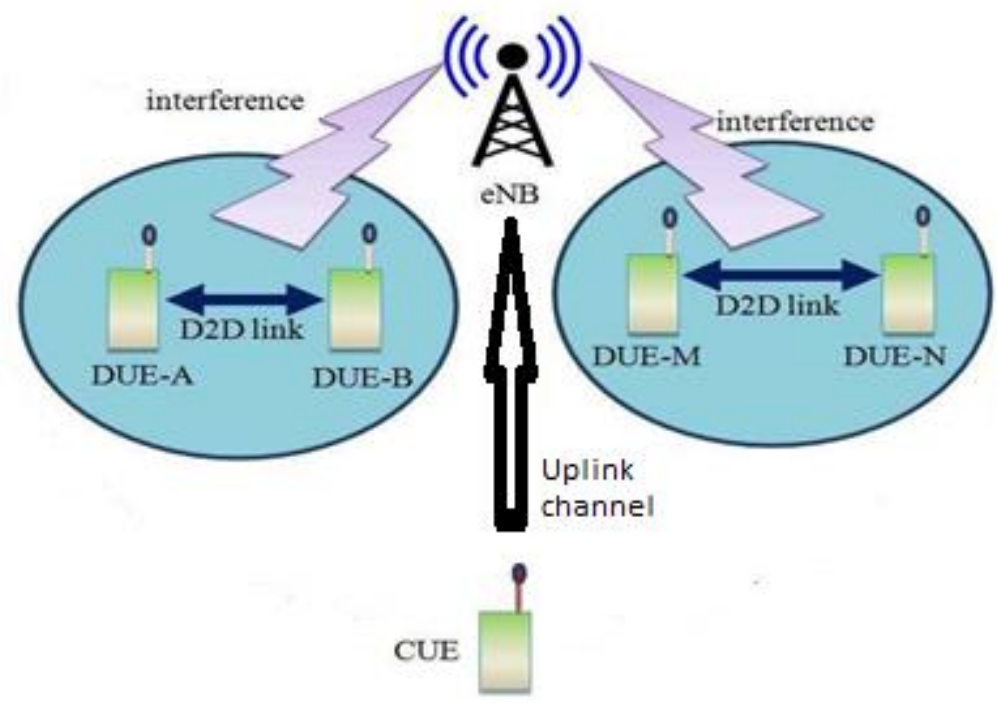

Fig.1. Interference problems between D2D pairs and CUs

The two main handover schemes for mobility management in D2D communication are D2D-Aware Handover and D2D-Triggered Handover schemes [6]. D2D-Aware Handover technique helps to reduce the latency when two devices are moving from one location to another. We propose a method which greatly reduces handover latency. D2D-Triggered Handover is a method of clustering the members of a D2D group to reduce the network signaling overhead due to information exchange between the base stations.

The D2D control and communications during the DUE mobility between two different base stations is illustrated in Fig.2. Fig. 2(a) depicts a situation where D2D pair initially controlled by BS1.

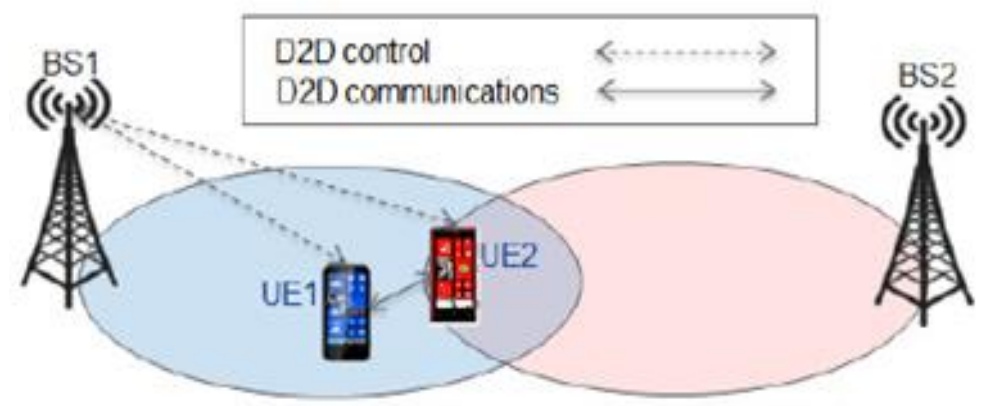

(a) 


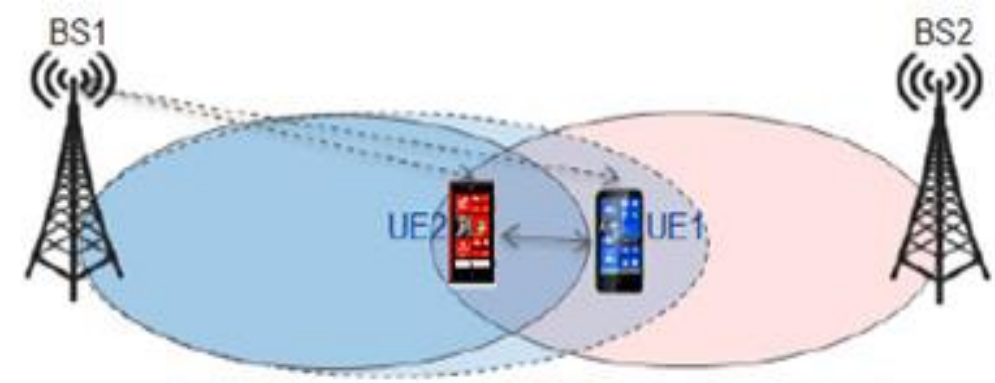

(b)

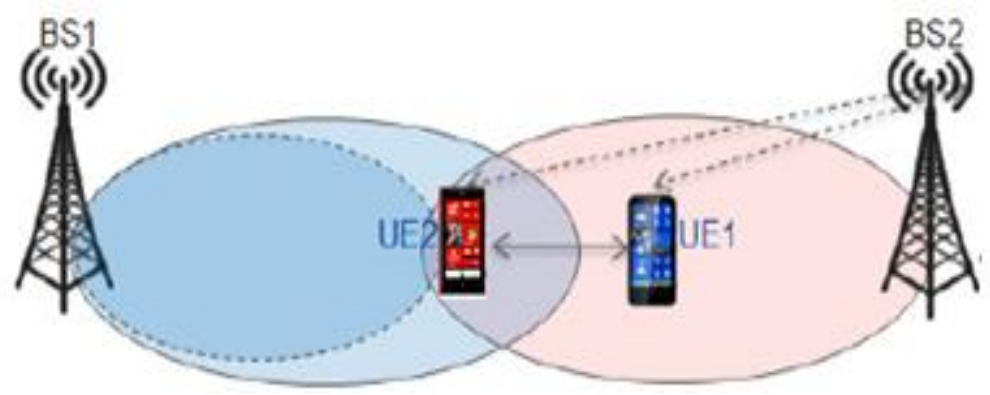

(c)

Fig.2. (a) Both UE1 and UE2 are controlled by BS1 (b) UE1 handover to BS2 is postponed until D2D conditions are fulfilled by both UEs (c) Handover to BS2 is executed when D2D conditions are fulfilled by both UEs

Fig.2(b) depicts a situation when UE1 move towards BS2. In this condition reducing the latency and signalling overhead becomes a challenging issue. We propose a solution in which BS1 postpones the handover till the signal quality of BS1 worse than a predefined D2D control condition to maintain the D2D control between two UEs. D2D control condition is set to the threshold value of signal-to-interference-plus-noise-ratio (SINR) i.e. $-6 \mathrm{~dB}$. Handover to BS2 is executed when D2D control conditions are fulfilled by both UE1 and UE2 as shown in Fig. 2(c).

\section{Interference and Mobility management}

\subsection{Interference mitigation}

Let $d_{j}$ be the D2D pair, $c_{i}$ be the cellular UE, $P^{c i}$ be the transmission power of UE, $\mathrm{P}^{\mathrm{dtj}}$ be the transmission power of D2D pair. Let the Gaussian noise variance to be $\sigma$ and assuming the D2D pair $\mathrm{d}_{\mathrm{j}}$ reuses the same base station, the Signal to Interference plus Noise Ratio (SINR) at the eNB according to [5] is

$$
\gamma_{c_{i} d_{j}}=\frac{P^{c_{i}} G^{c_{i}, e N B}}{\sigma+P^{d t} G^{d t j, e N B}}
$$

Where $\mathrm{G}^{\mathrm{dtj}, \mathrm{eNB}}$ is the channel gain between D2D transmitter and eNB, and $\mathrm{G}^{\mathrm{ci}, \mathrm{eNB}}$ is the channel gain between eNB and cellular UE $c_{i}$. 
Femto cell is the small low power base station. To find the Signal to Interference Noise Ratio (SINR) effect ' $\mathrm{d}$ ' is assumed to be the distance between the D2D user ' $\mathrm{m}$ ' and cellular base station ' $\mathrm{M}$ '. Let ' $D$ ' be the distance between the D2D users and is assumed to be $10 \mathrm{~m}$ to $100 \mathrm{~m}$.

The SINR of a macro cell user $m$ is given by [12]:

$$
\operatorname{SINR}_{m}=\frac{P_{M} G_{m, M}}{N_{o} \Delta_{f}+\sum_{M^{1}} P_{M^{1}} G_{m, M^{1}}+\sum_{F} P_{F} G_{m, F}}
$$

Where $\mathrm{P}_{\mathrm{M}}$ and $P_{M^{1}}$ are the transmit powers of the serving cell $\mathrm{M}$ and adjacent cell $\mathrm{M}^{1} \cdot \mathrm{G}_{\mathrm{m}, \mathrm{M}}{ }^{1}$ is the channel gain between the serving cell $\mathrm{m}$ and adjacent cell $\mathrm{M}^{1} . \mathrm{G}_{\mathrm{m}, \mathrm{F}}$ is the channel gain between the serving cell $\mathrm{m}$ and femto cell F. $P_{F}$ is the transmit power of adjacent femto cell $F$. $N_{o}$ is the white noise power spectral density. $\Delta_{f}$ is the subcarrier spacing.

The DUE SINR from [14] is given by

$$
\operatorname{SINR}_{D U E^{K}}=\frac{P_{D U E^{K}}\left|h_{D U E^{K}}\right|^{2}}{I E I^{K}+\sigma^{2}}
$$

Where $\mathrm{P}_{\mathrm{DUE}}{ }^{\mathrm{K}}$ is the power transmitted by the device to device user equipment DUE which is calculated by using the equation

$$
P_{D U E^{K}}=\min \left\{P_{\max }, P 0+\alpha P L\right\}
$$

Where $\mathrm{P}_{\max }$ is the maximum transmit power of the $\mathrm{D} 2 \mathrm{D}$ user. $\mathrm{P} 0$ is the uplink device transmission power during noise condition of the receiver. $\alpha$ is the parameter which represents path loss effect and the value of it is set as $\alpha=\{0,0.1,0.2,0.3, \ldots \ldots \ldots . .0 .9,1\}$. PL is the downlink path-loss effect obtained from each user. $\left|h_{D U E^{K}}\right|^{2}$ is the gain of the target D2D pair and $\mathrm{IEI}^{\mathrm{k}}$ is the In-band Emission Interference from the cellular user equipments.

Greedy algorithm is used to find the shortest path between the devices. The UE makes routing decisions based on the distance of the other UE either through BS or direct D2D communication. Table 1 gives the parameters considered for SINR.

Table 1. Parameters for SINR

\begin{tabular}{ll}
\hline Parameter & Value \\
\hline Subcarrier spacing & $15 \mathrm{KHz}$ \\
Number of Users N & 15 \\
Base station transmit power & $46 \mathrm{dBm}$ \\
Propagation Path Loss $\lambda$ & 0.8 \\
Source D2D UE transmit frequency $m$ & $2.1 \mathrm{GHz}$ \\
Neighbor Cell Power Interference & $-10.3 \mathrm{DBM}$ \\
Capacity of Cell M & $10^{5} \mathrm{BPS}$ \\
Destination D2D UE m' & $19(\mathrm{BS}$ number $)$ \\
\hline
\end{tabular}


In this shortest path greedy algorithm each D2D UE knows its location through its global position system. The Greedy algorithm uses the following steps:

i. The source UE makes communication request from the destination UE when the distance between them is in the region of proximity. Then the source UE performs D2D communication with the destination UE with the proper authentication.

ii. If the destination user equipment is not in the range of source user equipment, source user equipment calculates the shortest path and requests nearby BS to transfer the call to the destination user equipment.

iii. The source user equipment sends the data packet to the relay which is the close to the destination user equipment.

\section{Algorithm 1: Greedy Algorithm for Interference mitigation}

1. procedure probability $\mathrm{P}_{\mathrm{SPR}}\left(\mathrm{m}, \mathrm{M}, \mathrm{D}, \mathrm{d}_{\mathrm{j}}\right)$

2: if $\gamma_{\mathrm{cidi}} \geq \mathrm{D}$ then $\%$ the first hop SINR requirement

3: $\operatorname{SINR}_{m}=P_{M} G_{m, M} /\left(N_{o} \Delta_{f}+\sum_{M^{1}} P_{M 1} G_{m, M^{1}}+\sum_{F} P_{F} G_{m, F}\right)$

4: while destination node not reached do

5: Power transmitted DUE $P_{D U E^{K}}=\min \left\{P_{\max }, P O+\alpha P L\right\}$

\section{6: end while}

\section{7: end if}

8: The DUE SINR from [8] is given by $\operatorname{SINR}_{D U E^{K}}=\left(P_{D U E^{K}}\left|h_{D U E^{K}}\right|^{2}\right) /\left(I E I^{K}+\sigma^{2}\right)$

\section{9: end procedure}

\subsection{Handover analysis for mobility management}

The overall latency according to [7] is equal to

$$
T_{\text {scan }}+T_{\text {auth }}+T_{r e}-a s s+T P B U+T R A
$$

However, according to [8] MN's profile contains the MN-ID placed in nMAG, which authenticated the MN already. Hence, authentication delay can be removed during handover [9]. Hence overall latency according to $[6]$ is

$$
\operatorname{Total}_{H D}=T_{\text {scan }}+T_{r e}-a s s+T P B U+T P B A
$$

Since the mobile node keeps scanning for the surrounding networks existence continuously scanning delay cannot be removed. Hence in our proposed approach scanning delay considered. The proposed approach comparison with the standard approach is as shown in Fig. 3. First, the source user equipment calculates the distance of destination user equipment and checks for resources after authentication. If the source user equipment distance is in the line of sight communication, the source user equipment directly communicates with the destination user equipment by performing IP assignment and Registration. If the source user equipment distance is not in the line of sight communication, then the source user equipment checks for handover requirement and handover takes place when the destination user equipment is not under the same network. If both source and destination user equipments are under the same network and the distance between them is not in the line of sight communication, the BS establishes call setup process between them. In the proposed approach it is shown that the pre-authentication, pre-channel assignment, pre-IP assignment and pre- 
resource allocation processes will be completed before call setup when the distance between them is in the line of sight communication. It is also shown that the handover delay is greatly reduced due to pre-authentication, pre-channel assignment and pre-IP assignment.
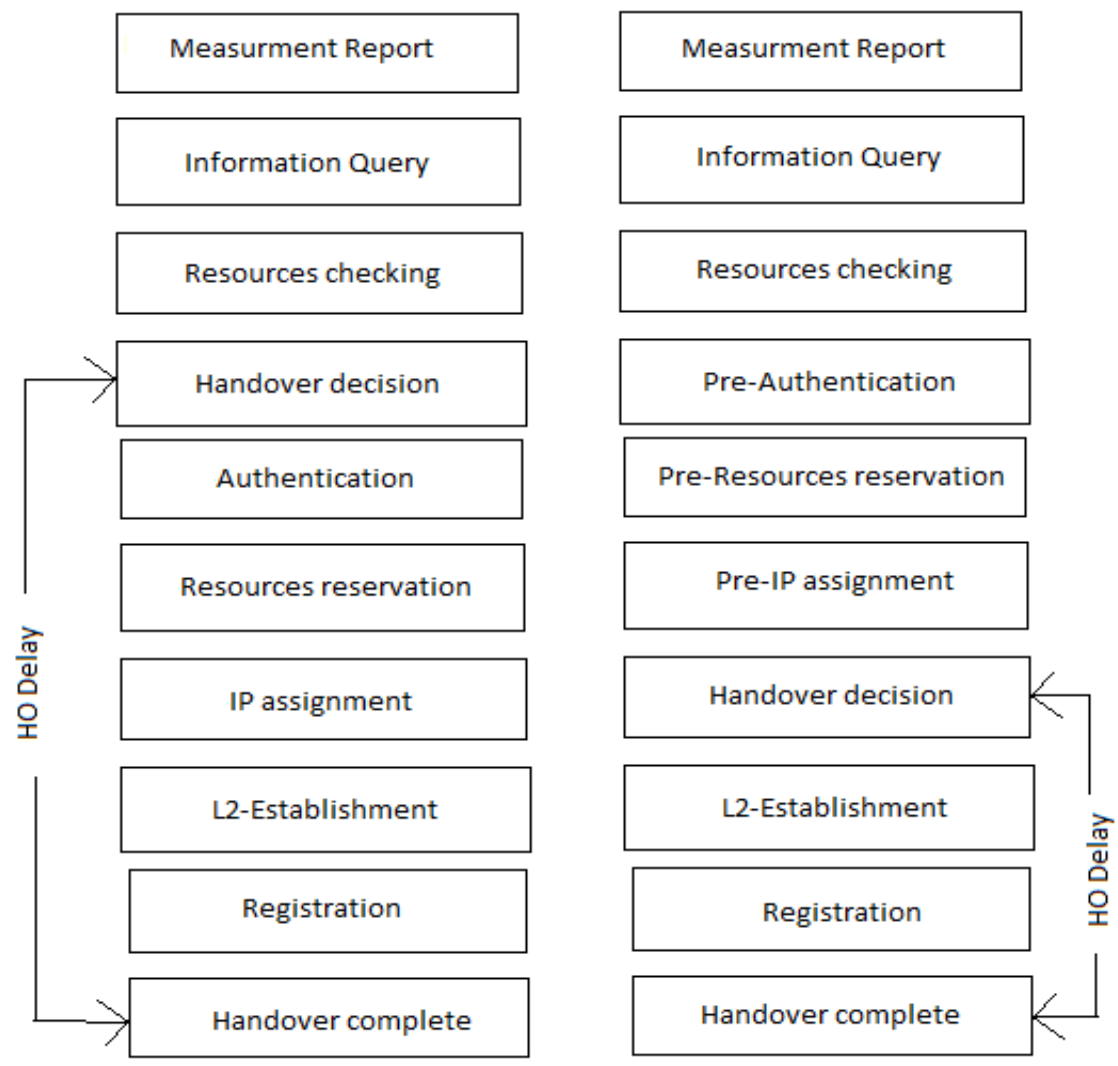

Fig.3. Comparison of proposed approach with the standard approach

Thus the overall HL of our approach is expressed as

$$
H L_{\text {proposed }}=T_{\text {scan }}+T_{r e}-a s s+T P B U+T R A
$$

\section{Simulation Results}

This section gives simulation results for the interference mitigation and handover delay. The Signal to Interference Noise Ratio results for the proposed system with increased D2D pairs is shown in Fig.4. 


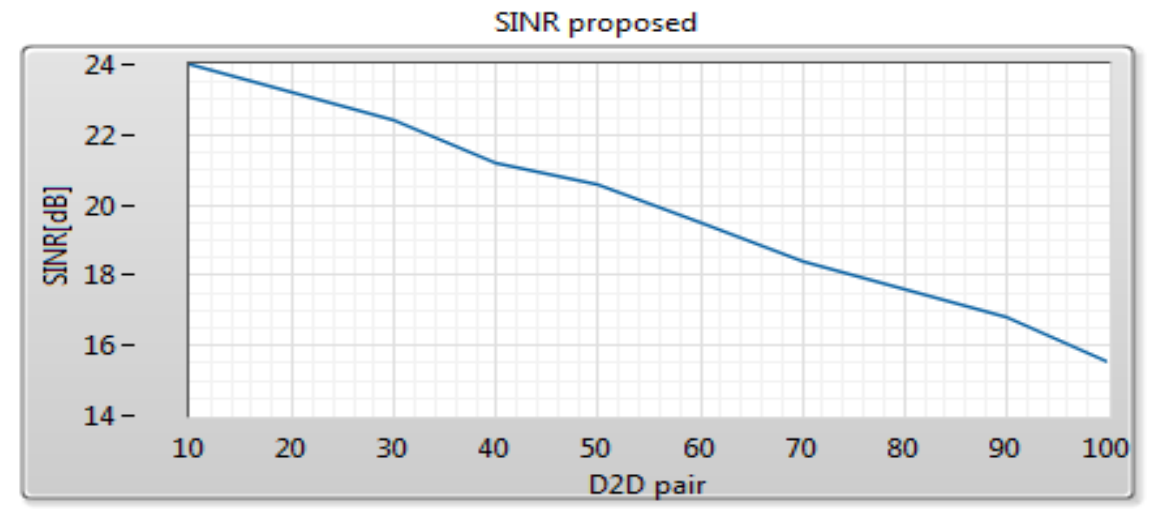

Fig.4. SINR values with increased D2D pairs (SINR-Proposed)

The maximum and minimum transmit powers of single eNB are assumed to be $46 \mathrm{dBm}$ and $10 \mathrm{dBm}$. The transmit power of eNB is set to be $34 \mathrm{dBm}$. The cellular network cell radius is set to be 2000 meters. The Cellular user equipment and device to device user equipment pairs are randomly deployed. The transmitting range of D2D pair is considered to be 1000 meters. The Signal to Interference Noise Ratio results for the conventional approach is shown in Fig.5.

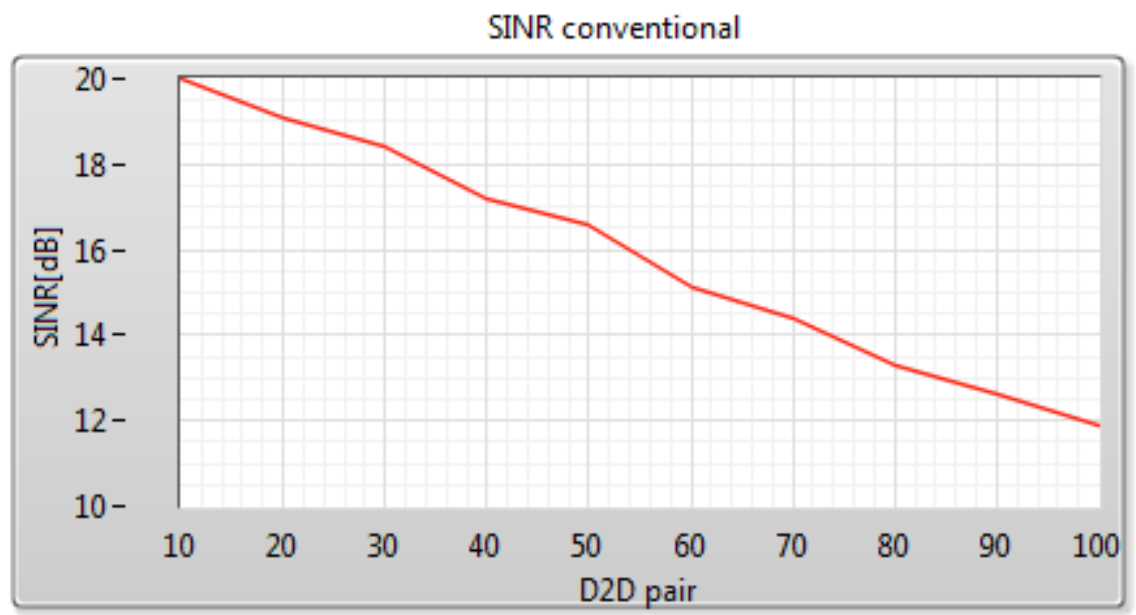

Fig.5. SINR values with increased D2D pairs (SINR-Conventional)

From the results it is observed that for 10 D2D pairs an SINR of $24 \mathrm{~dB}$ is obtained from the proposed system and it is $20 \mathrm{~dB}$ in the conventional method. Hence there is a increase in SINR by $4 \mathrm{~dB}$ from the proposed method. Simulation is performed under LabVIEW platform. Table 2 gives the parameters considered for SINR. 
Table 2. Simulation parameters

\begin{tabular}{ll}
\hline Parameter & Value \\
\hline Maximum Transmit Power of eNB & $46 \mathrm{dBm}$ \\
Minimum Transmit Power of $e N B$ & $10 \mathrm{dBm}$ \\
Pathloss model for D2D users & $38.46+20 \log _{10}\left(d_{d}\right) \mathrm{dB}$ \\
downlink path-loss $\left(\mathrm{P}_{\mathrm{L}}\right)$ & $15.3 \mathrm{~dB}$ \\
Max allowed transmit power (Pmax) & $200 \mathrm{~mW}$ \\
device specific transmission power $($ Po $)$ & $100 \mathrm{~mW}$ \\
System Bandwidth $(B)$ & $15 \mathrm{kHz}$ \\
SINR threshold $\xi(\mathrm{BS}$ antenna height) & $45 \mathrm{~m}$ \\
D2D pair distance & $10 \mathrm{~m}$ \\
Uplink bandwidth & $5 \mathrm{MHz}$ \\
\hline
\end{tabular}

For our proposed approach of mobility management considering scanning delays the time required for overall handover latency is HL-proposed $=$ Tscan + Tre - ass + TPBU + TRA $=0.6+25+11+30=66.6 \mathrm{~ms}$. Fig. 6 illustrates the Handover Latency versus MN velocity of the existing approach proposed in [6]. It is noted that the Handover delay for the MN velocity of $30 \mathrm{~m} / \mathrm{s}$ is $145 \mathrm{~ms}$ in the existing approach.

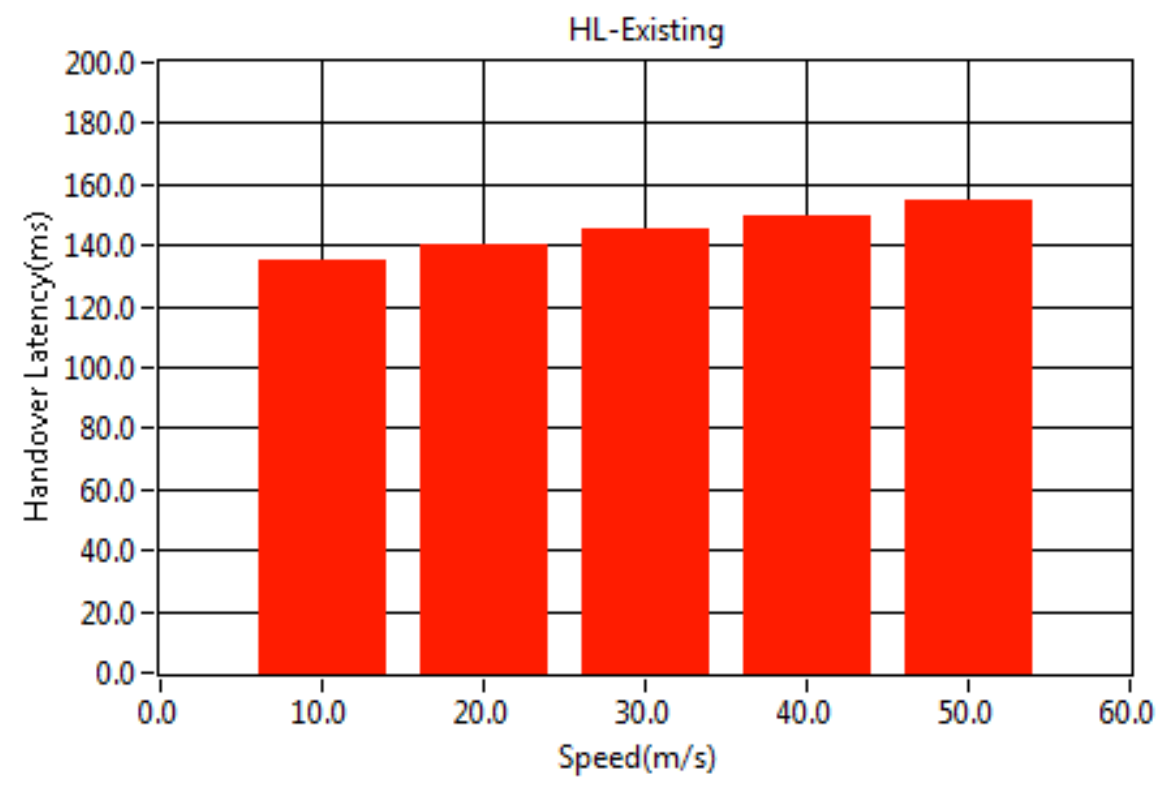

Fig.6. Handover latency versus MN velocity (Existing)

Fig.7 illustrates the Handover Latency versus MN velocity of the proposed approach. For simulation the velocity of $\mathrm{MN}$ is assumed from $10 \mathrm{~m} / \mathrm{s}$ to $50 \mathrm{~m} / \mathrm{s}$. It is observed that the Handover delay for the MN velocity of $30 \mathrm{~m} / \mathrm{s}$ is $66.6 \mathrm{~ms}$ in the proposed approach. Hence our proposed greatly helps in reducing delays during handover. 


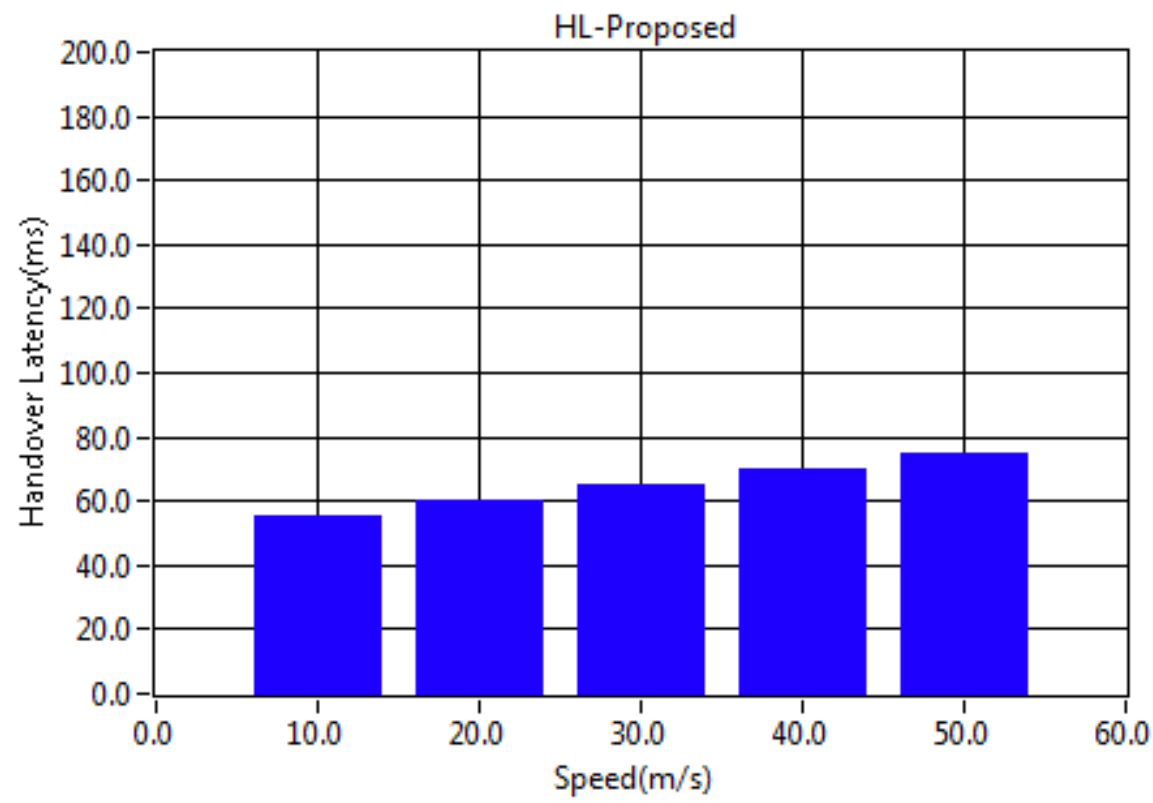

Fig.7. Handover latency versus MN velocity (Proposed)

The details of the proposed approach with other approaches are shown in Table 3.

Table 3. Performance evaluation parameters

\begin{tabular}{llll}
\hline Parameter & & & Value \\
\hline Tscan & 0.6 & 0.6 & 0.6 \\
Tauth & 0.6 & 0.6 & \\
Tre-ass & 250 & 25 & 25 \\
TMN & 0.1 & - & - \\
TDAD & 1070 & - & - \\
TPBU & 11 & 11 & 11 \\
TPBA & 11 & - & - \\
TRA & 30 & 30 & 30 \\
Total & $1373.3 \mathrm{~ms}$ & $67.2 \mathrm{~ms}$ & $66.6 \mathrm{~ms}$ \\
\hline
\end{tabular}

\section{Conclusions}

Interference minimization and mobility management techniques are addressed in this paper for device to device communication in LTE-A networks. The power control techniques are shown to obtain improvements in SINR values. An improvement by $4 \mathrm{~dB}$ in SINR values is observed. For simulation we set the eNB transmit power to be $34 \mathrm{dBm}$ and the network cell radius to be 2000 meters.

In our proposed approach of mobility management a solution is obtained to minimize the handover latency. We studied the standard methods and the work shown by different authors to reduce handover latency. The time required for overall handover latency is calculated considering the requirements of authentication and scanning parameters when a DUE moving from one BS coverage area to the other. In the proposed approach 
we have shown that the time required for overall handover latency to be $66.6 \mathrm{~ms}$ for the MN velocity of $30 \mathrm{~m} / \mathrm{s}$ and it is $145 \mathrm{~ms}$ in the existing approach. Hence our proposed greatly helps in reducing handover delays when the MN shifts from one cell to the other.

\section{References}

[1] Misfa Susanto, Helmy Fitriawan, Andri Abadi, Herlinawati, "On the Reduction of Interference Effect Using Power Control for Device-to-Device Communication Underlying Cellular Communication Network", International Conference on Electrical Engineering and Computer Science (ICECOS) 2017, pp. 28-32. 2017.

[2] Devarani Devi Ningombam and Seokjoo Shin, "Radio Resource Allocation and Power Control Scheme to Mitigate Interference in Device-to-Device Communications Underlaying LTE-A Uplink Cellular Networks", IEEE International Conference on Information and Communication Technology Convergence(ICTC), pp. 961-963, 2017.

[3] Zekun Zhang, Rose Qingyang Hu, Yi Qian, "D2D Communication Underlay in Uplink Cellular Networks with Distance Based Power Control ", IEEE International Conference on Communication, pp. 1-5, 2016.

[4] Selma SBIT,Mohamed Bechir DADI and Belgacem CHIBANI, "SINR and Throughput enhancement in LTE-Advanced", 16th international conference on Sciences and Techniques of Automatic control \& computer engineering - STA'2015, pp.779-782, 2015.

[5] MD. Yeakub Hassan1, Faisal Hussain1, MD. Sakhawat Hossen1, "Interference Minimization in D2D Communication Underlying Cellular Networks", IEEE Access, Vol. 5, pp.22471-22484, 2017.

[6] Nouri Omheni1, Imen Bouabidi1, Amina Gharsallah1, Faouzi Zarai1, Mohammad S. Obaidat2, "Smart mobility management in 5G heterogeneous networks", IET Networks Research Article, Vol. 7, pp. 119$128,2018$.

[7] Kim, I., Jung, Y.C., Kim, Y.T, "Low latency proactive handover scheme for proxy MIPv6 with MIH", 11th Asia-Pacific Symp. Network Operations and Management (APNOMS): Challenges for Next Generation Network Operations and Service Management, vol. 5297, pp. 344-353, 2008.

[8] Shouman Barua, Robin Braun, "Mobility Management of D2D Communication for the 5G Cellular Network System: A Study and Result", 2017 17th International Symposium on Communications and Information Technologies (ISCIT), pp. 119-128, 2018.

[9] Md Mehedi Hasan, Sungoh Kwon, "Adaptive Mobility Load Balancing Algorithm for LTE Small-Cell Networks", IEEE transactions on wireless communications, Vol. 17, No. 4, pp. 2205-2217, April 2018.

[10] A. Elakkiya, P.Selvaraj, "QoS Based IP Mobility Management Scheme for the Next Generation SDNLTE Network", Proceedings of the Second International Conference on Inventive Systems and Control (ICISC 2018), pp. 1355-1360, 2018.

[11] Xiaomeng Li, Yuan Ren, Hui Gao, and Tiejun Lv, "Opportunistic Interference Alignment Approach in Device-to-Device Communications Underlaying Cellular Networks", 2016 24th European Signal Processing Conference (EUSIPCO), pp. 1838-1842, 2016.

[12] Selma SBIT,Mohamed Bechir DADI and Belgacem CHIBANI, "SINR and Throughput enhancement in LTE-Advanced", 16th international conference on Sciences and Techniques of Automatic control \& computer engineering - STA'2015, pp.779-782, 2015.

[13] Chungang Yang, Jiandong Li, Prabodini Semasinghe, "Distributed Interference and Energy-Aware Power Control for Ultra-Dense D2D Networks," IEEE Trans. Wireless Commun., vol. 16, no. 2, pp. 1205-1217, 2017.

[14] Ravindra S, Dr. Siddesh G K, "Power Control in LTE-Advanced Networksfor Device to Device Communication",International Journal of Advanced Research in Electrical, Electronics and Instrumentation, Engineering, ISSN (Print) : 2320 - 3765, ISSN (Online): 2278 - 8875, Volume 6, Issue 4, PP:2219-2223, April 2017 
[15] T Gopi Sai Krishna, Ramarakula Madhu, "A Utility Function based Uplink Power Control Algorithm in LTE-Advanced Networks," International Conference on Communication and Signal Processing, vol. 16, pp. 705-708, 2016.

\section{Authors' Profiles}

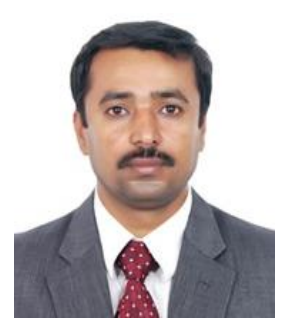

Ravindra S (born July 1 1980) is a research scholar. He has done his engineering in the field of Telecommunication engineering and M.Tech in the field of VLSI and embedded systems. He is pursuing his PhD on Qos based D2D Wireless communication in LTE-A networks.

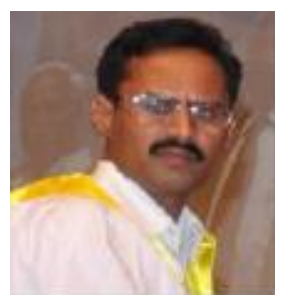

Dr. Siddesh G K(born July 10 1974) presently working as Professor and HOD in Electronics \& Communication Engineering department of JSSATE Bangalore. He has done his Phd in the field of Ad-Hoc wireless communication. Siddesh G K is awarded with "Best Professor in Electronics \& Communication Engineering" from Dewang Mehta National Education Award on 20 ${ }^{\text {th }}$ September 2017 at Vivanta Taj, Bengaluru.

How to cite this paper: Ravindra S, Siddesh G K, "Interference Mitigation and Mobility Management for D2D Communication in LTE-A Networks", International Journal of Wireless and Microwave Technologies(IJWMT), Vol.9, No.2, pp. 20-31, 2019.DOI: 10.5815/ijwmt.2019.02.03 